\title{
Intraocular pressure and pupillary responses in patients with diabetes mellitus
}

\author{
M.R. Al-Sereiti, ${ }^{*}$ P. Turner and E.A.M. Gale ${ }^{1}$
}

Department of Clinical Pharmacology and ${ }^{1}$ Department of Diabetes and Immunogenetics, St Bartholomew's Hospital, London EC1A 7BE, UK

Summary: Intraocular pressure (IOP) and the mydriatic responses to darkness in 38 diabetic patients have been compared with those of a matched control group of normal subjects. Overall, the diabetic patients had a significantly higher mean IOP than the control group, but this difference was not found in a sub-group of 13 diabetic patients with evidence of autonomic neuropathy. Those diabetic patients with $\theta$ evidence of autonomic neuropathy had a smaller mydriatic response to darkness than the control group, and this test may usefully be added to standard tests of autonomic function in the diagnosis of autonomic neuropathy.

\section{Introduction}

There appears to be a relationship between diabetes and raised intraocular pressure (IOP). It has been reported that abnormal oral glucose tolerance tests and evidence of autonomic neuropathy are more prevalent in patients with ocular hypertension and glaucoma than in the normal population. ${ }^{1,2}$ The authors suggested that glaucoma may be a physical sign of diabetes mellitus, although another study ${ }^{3}$ could find no difference in the glucose tolerance of patients with closed-angle glaucoma compared with normal subjects. It is also well documented that glaucoma is more prevalent in diabetic patients than in normal control groups. ${ }^{4,5}$ Autonomic neuropathy is a specific complication of diabetes mellitus, ${ }^{6}$ and the pupils of diabetic patients with autonomic neuropathy dilate less in darkness than those of normal controls. ${ }^{7}$

We have compared IOP and the mydriatic response to darkness in a group of diabetic patients with those of a matched control group of normal subjects.

\section{Methods}

Thirty eight patients ( 29 males) from the diabetic clinic of this hospital, already diagnosed as having type I or type II diabetes mellitus, volunteered for the study and were screened for evidence of autonomic neuropathy with the criteria that one or more of the following tests were positive. ${ }^{8}$

Correspondence: Professor P. Turner, M.D., B.Sc., F.R.C.P., F.F.P.M.

*Present address: Faculty of Medicine, Tripoli, Libya Accepted: 29 October 1990
1. Heart rate variation during deep breathing, calculated from a continuous electrocardio 3 gram (ECG) tracing recorded during 6 succes $\stackrel{\frac{0}{3}}{\frac{1}{3}}$ sive deep breaths.

2. Immediate heart rate response to standifge using a continuous ECG tracing.

3. Blood pressure response to standing, usi the Dinamap vital signs monitor, 1846.

4. Blood pressure response to sustained hand grip, using a sphygmomanometer with its cuff replaced by an inflatable bag as a handgrip dynamometer.

IOP was measured by non-contact tonometry, $, 9,10 \overrightarrow{\vec{D}}$ which has a mean intra-individual within-time between days coefficient of variation of $9.5 \%$. 毠 Pupillary responses to darkness, expressed as the ratio of pupil:iris diameter (\%) after 1 minute in total darkness, were measured by photography. using a Polaroid CU-5 Close-Up hand camera with a built-in flash, 1:3 ratio multiplier and frame. Preliminary studies had shown that the mean intra-individual within-time between days coefficient of variation of the method was $4.5 \% .^{11}$

IOP measurements and pupil:iris diameter ratioso $(\mathbf{P} / \mathbf{I} \%)$ of the diabetic subjects were compared with those of 38 normal subjects matched for age, sexo and eye colour, using Student's unpaired $t$-test. Then study was approved by the local ethics committee, N and all subjects gave their consent to participate.

\section{Results}

Mean ( \pm s.d.) values of age, IOP measurement and ${ }^{0}$ $\mathbf{P} / \mathbf{I} \%$ ratios are shown in Tables $I$ and II. The mean IOP values of the diabetic patients were $\frac{\text { Pे }}{\mathrm{O}}$ 
Table I Mean ( \pm s.d.) age, IOP and pupil/iris ratio (\%) of 38 diabetic patients, and normal controls matched for age, sex and eye colour

\begin{tabular}{lccccc}
\hline & & \multicolumn{4}{c}{ IOP mmHg } \\
& $n$ & Age $(y)$ & Right & Left & $P / I^{\%}$ \\
\hline Diabetic patients & 38 & 40 & $15.3^{*}$ & $15.3^{*}$ & 52.26 \\
& & $(15)$ & $(3.4)$ & $(3.3)$ & $(9.24)$ \\
Normal controls & 38 & 41 & 13.3 & 13.2 & 55.78 \\
& & $(14)$ & $(4.3)$ & $(4.6)$ & $(9.03)$ \\
\end{tabular}

${ }^{*} P<0.05$ compared with controls.

Table II Mean ( \pm s.d.) age, IOP and pupil/iris ratio (\%) of 13 diabetic patients with autonomic neuropathy and their normal controls matched for age, sex and eye colour

\begin{tabular}{lccccc}
\hline & & \multicolumn{4}{c}{ IOP mmHg } \\
& $n$ & Age (y) & Right & Left & $P / I^{\%}$ \\
\hline Diabetic patients & 13 & 49 & 15.4 & 15.6 & $45.05^{*}$ \\
$\begin{array}{l}\text { with autonomic } \\
\text { neuropathy }\end{array}$ & & $(13)$ & $(3.8)$ & $(4.0)$ & $(8.46)$ \\
Normal controls & 13 & 49 & 15.5 & 15.2 & 52.75 \\
& & $(13)$ & $(4.5)$ & $(5.0)$ & $(9.69)$ \\
\hline
\end{tabular}

${ }^{*} P<0.05$ compared with controls.

significantly higher than those of their matched controls $(P<0.05)$ in both right and left eyes. The IOP values of the patients with evidence of autonomic neuropathy (13 patients, 9 males) were not significantly different from those of their matched controls. The mean $P / I$ ratio of the diabetic patients was not significantly different from the controls overall, but the mean ratio of the 13 patients with evidence of autonomic neuropathy

\section{References}

1. Clark, C.V. \& Mapstone, R. Autonomic neuropathy in ocular hypertension. Lancet 1985, ii: 185-187.

2. Mapstone, R. \& Clark, C.V. Prevalence of diabetes in glaucoma. $\mathrm{Br}$ Med J 1985, 291: 93-95.

3. Clarke, M. \& Jagger, J. Is acute glaucoma a symptom of diabetes? Br J Ophthalmol 1986, 70: 877-878.

4. Becker, B. Diabetes mellitus and primary open-angle glaucoma. Am J Ophthalmol 1971, 71: 1-16.

5. Nielsen, N.V. The prevalence of glaucoma and ocular hypertension in type 1 and 2 diabetes mellitus: an epidemiological study of diabetes mellitus on the island of Falster, Denmark. Acta Ophthalmol 1983, 61: 662-672.

6. Watkins, P.J. \& Edmonds, M.E. Clinical presentation of diabetic autonomic failure. In: Bannister, R. (ed.) Autonomic Failure. Oxford Medical Publications, Oxford, 1983, pp. 337-370. was significantly lower $(P<0.05)$ than that of their matched controls.

\section{Discussion}

These results show that diabetic patients with no previous history of ocular hypertension had a significantly higher mean IOP than a normal control group matched for age, sex and eye colour. This difference was not found, however, in a sub-group of patients with evidence of autonomic neuropathy. These findings run contrary to the conclusion of other investigators that ocular hypertension is more prevalent in diabetics with, rather than without, evidence of autonomic neuropathy. ${ }^{1,2}$ and this question, therefore, requires further investigation in larger numbers of subjects. However, since the prevalence of glaucoma is increased in diabetic patients, ${ }^{4,5}$ our observations of increased IOP in a group of patients taken from a diabetic clinic, would suggest that the IOP of diabetic patients should be measured routinely at regular intervals to detect the development of ocular hypertension at an early stage. The non-contact tonometer provides a convenient method for such a screening procedure, as it requires no topical anaesthesia or contact with the eye, and is quickly carried out.

Our results also confirm the observations of other investigators ${ }^{7}$ that diabetic patients with other evidence of autonomic neuropathy have a smaller mydriatic reaction to darkness than a control group. This test may usefully be added to standard tests of autonomic function in the diagnosis of autonomic neuropathy. It is possible that the normal IOP in these patients is associated with their reduced $P / I$ ratio, and the increased aqueous drainage accompanying it.

7. Smith, S.Q. \& Dewhirst, R.R. A simple diagnostic test for pupillary abnormality in diabetic autonomic neuropathy. Diabetic Med 1986, 3: 38-41.

8. Ewing, D.J. \& Clark, B.F. Diagnosis and management of diabetic autonomic neuropathy. $\mathrm{Br} \mathrm{Med} J$ 1982, 285: 916-918.

9. Grolman, B. A new tonometer system. Am J Optometry Physiol Opt 1972, 49: 646-650.

10. Shields, M.B. The non-contact tonometer - its value and limitations. Surv Ophthalmol 1980, 24: 211-219.

11. Al-Sereiti, M.R. The effects of some dopamine receptor agonists, beta-adrenoceptor antagonists and angiotensinconverting enzyme inhibitors on intraocular pressure and pupil diameter in man. Ph.D. Thesis, University of London, 1989, p. 92, 100. 